\title{
Modelo teórico-metodológico baseado na Teoria de Redes Complexas para análise da oferta potencial dos serviços de saúde
}

\author{
Theoretical-methodological model based on Complex Network \\ Theory for the analysis of the potential provision of health services
}

Deivson Rayner Teixeira da Costa (https://orcid.org/0000-0001-6260-0085) ${ }^{1}$

Jorge Otávio Maia Barreto (https://orcid.org/0000-0002-7648-0472) ${ }^{2}$

Ricardo Barros Sampaio (https://orcid.org/0000-0002-6989-8555) ${ }^{2}$

${ }^{1}$ Universidade de Brasília. Campus Universitário Darcy Ribeiro s/n, Asa Norte. 70910-900 Brasília DF Brasil.

deivsonrayner@gmail.com

${ }^{2}$ Fundação Oswaldo Cruz. Brasília DF Brasil.

\begin{abstract}
The scope of this article is to present a theoretical-methodological model as the basis for the analysis of the potential provision of Brazilian public health services (SUS) using an approach based on complex network theory. The model sought to analyze the potential provision of health services through the relationship of Euclidean distance between census sectors and services offered by health facilities, as defined in the National Register of Health Establishments (CNES). Two types of metrics are proposed for the analysis of the relationship network: the average distance of health services to the census sectors and the importance of the health unit in the potential provision of each type of service based on the centrality and availability of this service. To demonstrate the application of the model, a network was built based in the Metropolitan Area of Brasilia, including the Federal District and 12 surrounding municipalities. The model was able to map and analyze CNES data with census sectors defined in the CENSUS, opening up the possibility of constructing new perspectives of analysis in the understanding of the potential distribution of health resources and services according to socioeconomic variables, as well as an important evidence-based management tool.
\end{abstract}

Key words Access to health services, Health care, Public Health, Data science, Complex networks
Resumo $O$ objetivo deste artigo é apresentar um modelo teórico-metodológico como base para a análise da oferta potencial de serviços de saúde pública brasileira a partir de uma abordagem baseada na teoria de redes complexas. O modelo analisou a oferta potencial de serviços de saúde através da relação da distância euclidiana entre setores censitários e serviços/classificadores oferecidos pelos estabelecimentos de saúde, conforme definidos no Cadastro Nacional de Estabelecimentos de Saúde (CNES). São propostos dois tipos de métrica para análise dos relacionamentos: a distância média dos serviços de saúde para com os setores censitários e a importância da unidade de saúde na oferta potencial de cada tipo de serviço com base na centralidade e na disponibilidade deste serviço. Para demonstrar a aplicação do modelo foi construída uma rede com base na Área Metropolitana de Brasília, incluindo o Distrito Federal e 12 municípios do entorno. O modelo demostrou-se capaz de mapear e analisar os dados do CNES com os setores censitários definidos no Censo, abrindo a possibilidade de construção de novas óticas de análise na compreensão da distribuição potencial de recursos e serviços em função de variáveis socioeconômicas, bem como, uma importante ferramenta de gestão informada por evidências.

Palavras-chave Acesso aos Serviços de Saúde, Assistência à Saúde, Saúde 


\section{Introdução}

O Sistema Único de Saúde (SUS) consiste em uma estrutura baseada em redes de atenção, com características de unicidade e descentralização, e a distribuição de seus serviços de saúde é resultado da articulação organizada entre diferentes níveis de atenção, desde os serviços de atenção básica e até os de alta complexidade1. Embora esta organização seja hierarquizada em função da complexidade, estrutura-se na forma de redes integradas, tendo como foco uma população e territórios definidos. É papel desta rede, que tem a atenção básica como porta de entrada, buscar uma ampla compreensão das necessidades e preferências da população e determinar a oferta de serviços e ações de saúde. Desta maneira, a atenção básica desempenha um importante papel como porta de entrada e primeiro contato com a rede de saúde, e como articuladora dos demais níveis de atenção, atuando longitudinalmente e centrada na organização familiar e comunitária ${ }^{2}$.

No aspecto da regionalização, o SUS emprega as Regiões de Saúde como unidades de planejamento e articulação de recursos, bem como para estabelecer condições materiais para a integralidade da cobertura de serviços de saúde ${ }^{3}$. Contudo, ao utilizarmos este recorte espacial para analisar a distribuição dos serviços de saúde, o emprego das regiões de saúde pode induzir à perda de informação devido à agregação de diferentes condições sociais e econômicas presentes neste espaço geográfico, portanto, é oportuno a utilização de um recorte espacial mais localizado abrindo espaço para a possibilidade de desagregação das características socioeconômicas da região de saúde.

A distribuição da oferta de serviços de saúde em um território geográfico constitui uma das dimensões do acesso à saúde. Andersen ${ }^{4}$ utiliza o termo "acesso potencial" para definir a presença dos elementos necessários que permitem a execução de um determinado serviço. Desta maneira, $\mathrm{o}$ acesso potencial se caracteriza pela presença de fatores capacitantes e será diretamente afetado por barreiras que limitam a capacidade de uso destes serviços. Embora a presença ou ausência de um serviço em uma dada localidade não seja suficiente para se compreender de forma ampla as condições de acesso com equidade, esta variável é parte da fórmula. Portanto, ao avaliarmos acesso na perspectiva da barreira geográfica, o acesso com equidade será resultado da relação entre a distância geográfica do serviço em conjunto de variáveis que indicam a necessidade de acesso a este serviço ${ }^{4}$.
A perspectiva do acesso geográfico como um componente da avaliação do acesso dos serviços de saúde também foi abordada por Penchansky e Thomas ${ }^{5}$. Estes autores definiram "acessibilidade" sendo a relação da localização da oferta do serviço de saúde com a população potencial usuária deste serviço. Observa-se aqui elementos capacitantes, tais como recursos de transporte disponíveis e tempo de deslocamento até o estabelecimento de saúde, como componentes preditores da relação de acessibilidade. Esta acessibilidade, portanto, representa a cobertura quanto à disponibilidade do acesso físico aos serviços e recursos de saúde para a população, sendo entendido aqui como cobertura da acessibilidade e podendo contribuir para a compreensão da performance do sistema de saúde e potenciais falhas de sua cobertura ${ }^{6}$.

É relevante observar que, embora o conceito de 'acesso' seja multidimensional, a acessibilidade geográfica é, em si, um elemento importante para estudo isolado visto que a disposição geográfica do serviço é compreendida em vários estudos como fator determinante do uso de serviços de atenção básica ${ }^{6-8}$. Evidências demonstram que, quanto maior a barreira geográfica, menor a probabilidade de utilização de serviços de saúde, tais como o tratamento de câncer de mama e tratamento do alcoolismo, bem como maior é a incidência de problemas de saúde em crianças vivendo em áreas com falha na cobertura geográfica de serviços de atenção básica9 . Desta maneira, este artigo utiliza a noção de "acessibilidade" para representar a dimensão do "acesso geográfico" e "acesso potencial" como conceitos intercalados, representando o potencial de uso deste serviço em função da existência ou ausência de barreiras espaciais, apoiando-se nos modelos teóricos apresentados por Andersen ${ }^{4}$ e Penchansky e Thomas $^{5}$.

Considerando três premissas fundamentais: (A) O SUS estrutura-se como uma rede de atenção descentralizada que busca integrar diferentes níveis de complexidade, (B) o planejamento desta rede opera de forma descentralizada sobre uma região e população, $(\mathrm{C})$ a presença de um determinado serviço de saúde, independentemente do conhecimento de sua real necessidade é caracterizado pela oferta potencial deste serviço, fator predisponente para determinar o uso, e por seguinte, a equidade; o objetivo deste artigo é apresentar um modelo teórico-metodológico como base para análise da oferta potencial de serviços de saúde brasileira a partir da ótica do acesso geográfico, utilizando métricas oriundas da 
teoria de redes complexas e baseado na distribuição geográfica de setores censitários, permitindo um relacionamento direto aos dados censitários.

\section{Métodos}

Para o desenvolvimento do modelo proposto neste artigo utilizou-se os dados da Área Metropolitana de Brasília ${ }^{10}$ (AMB), espaço geográfico instituído pela Companhia de Planejamento do Distrito Federal (CODEPLAN) e composto pelo Distrito Federal e 12 municípios adjacentes pertencentes ao Goiás. Além disto, a modelagem utilizou como fontes secundárias as bases disponíveis publicamente pelo Cadastro Nacional de Estabelecimentos de Saúde ${ }^{11}$ (CNES) e o Censo ${ }^{12}$ de 2010, estes dados foram utilizados para modelagem dos estabelecimentos de saúde, serviços oferecidos e distribuição da população no território analisado.

Os setores censitários representam a unidade territorial estabelecida para controle cadastral. Cada setor é constituído por uma área territorial urbana ou rural com dimensão e número de domicílios determinados em função da organização do trabalho do recenseador ${ }^{12}$. Desta maneira, o setor censitário oferece um recorte territorial granular dentro do espaço definido pela região de saúde, permitindo oferecer uma relação mais próxima entre a localização de um serviço de saúde e a realidade socioeconômica presente neste setor.

A análise e construção conceitual do modelo baseou-se na Teoria de Redes Complexas, arcabouço teórico que sugere uma oportunidade no campo da compreensão das complexas relações entre a disposição dos setores censitários e a localização de serviços e recursos de saúde. A análise e modelagem sob esta ótica parte da premissa que existe relevância nas relações entre as entidades participantes do modelo, desta maneira, a unidade de análise não será individualmente cada unidade (ou seus agregados), mas sim o coletivo de suas relações ${ }^{13,14}$. Assim, em sua forma mais simples, uma rede é caracterizada por uma coleção de pontos (vértices) relacionados por ligações (links), permitindo o estudo de aspectos estruturais, fluxos de comunicação ou padrões de relacionamentos. Sendo, portanto, uma abstração de um sistema reduzido ao padrão de relacionamento entre suas entidades ${ }^{15}$.

- Os vértices da rede - representam, em geral, indivíduos, organizações ou comunidades, e no contexto deste artigo é representado pelo setor censitário e pelos estabelecimentos de saúde. A existência de distintos conjuntos de entidades em uma rede determinará seu número de modos. Neste modelo foi observado a existência de entidades do tipo Setor Censitário e do tipo Estabelecimento de Saúde, caracterizando a rede como uma estrutura bipartida. Uma característica de tais estruturas multimodais é a natureza dos relacionamentos entre conjuntos de entidades, vértices pertencentes ao mesmo conjunto não se relacionarão mutualmente, havendo, portanto, um particionamento da rede ${ }^{15}$. Estruturas com mais de um conjunto de entidades são tratadas por autores como Wilson ${ }^{16}$, Borgatti e Evertti ${ }^{17}$ onde abordam formulações estatísticas próprias para operação sobre estas estruturas.

- Os relacionamentos (links) da rede - Os relacionamentos operam como links entre os vértices representando empiricamente fluxos de transferência de recursos, materiais ou imateriais. Neste modelo, os relacionamentos foramestabelecidos pela relação potencial de acesso geográfico (distância euclidiana) entre um setor censitário e um, ou mais,estabelecimentos de saúde, sendo estes relacionamentos categorizados pelo tipo de serviço/classificador associado àcada relação. Boccaletti et al. ${ }^{18}$ discutem este tipo de arranjo multidimensional caracterizado por apresentar diversos canais de conectividade entre os vertices da rede, estrutura que aqui representa os direntes arranjos temáticos de análise.

Na Figura 1, é descrito o fluxo adotado para o processo de coleta e preparação destes dados e modelagem da rede.

O resultado final do processo de preparação dos dados é a malha-base da rede, composta pelo relacionamento de todos os setores censitários com pelo menos um serviço/classificador de cada tipo, disponível nos dados do CNES. O processo de transformação e manipulação dos dados utilizou scripts construídos na ferramenta RStudio ${ }^{19}$ e visualização da rede utilizando a ferramenta Gephi $^{20}$.

A preparação de dados e formação dos relacionamentos para construção da rede foram seguidos pelos seguintes passos:

a) Criação de uma base de dados de estabelecimentos de saúde pertencentes a $\mathrm{AMB}$ com base nos dados do CNES de dezembro de 2018 . Foram adicionados também os estabelecimentos de saúde pertencentes a municípios adjacentes aos 12 municípios do Goiás membros da AMB. Esta região foi denominada "zona de escape" da rede e tem o objetivo eliminar o viés descrito por Fortney et al. ${ }^{21}$ como "border cross problem" e que 


\begin{tabular}{|c|}
\hline Procedimentos \\
\hline Fontes de Dados \\
\hline Critérios de Exclusão \\
\hline Dados Resultantes \\
\hline
\end{tabular}
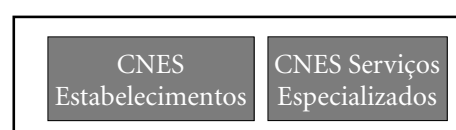

Arquivo DBF CNES-Dez/18

Seleção de Amostra Geográfica com base na Área Metropolitana de Brasília

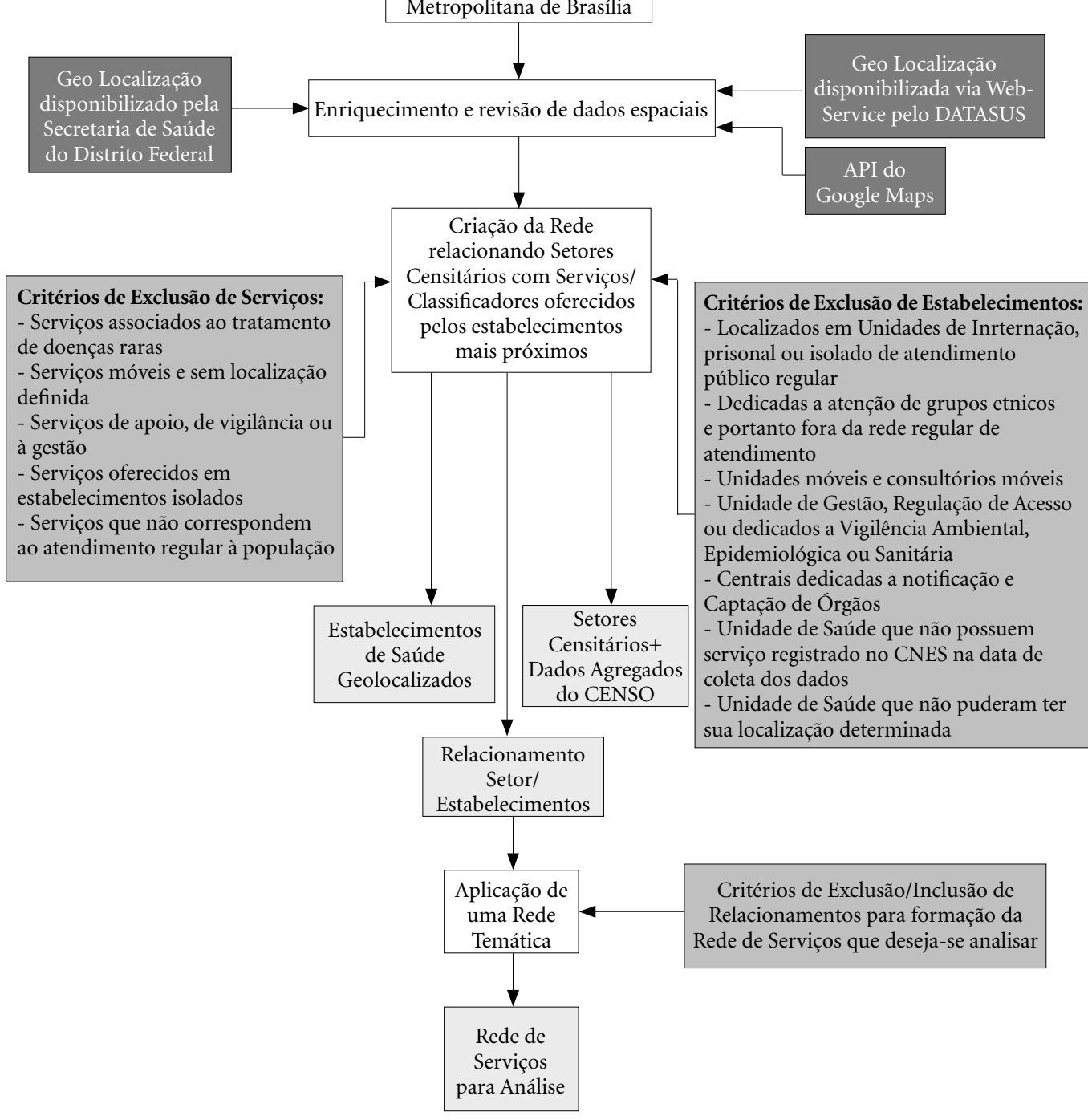

Figura 1. Diagrama do processo de coleta de dados e montagem da rede.

Fonte: Elaborado pelos autores, 2019.

é caracterizado pela baixa acessibilidade de espaços geográficos localizados na borda da região estudada.
CENSO 2010-

Dados Agregados

por Setor Censitário

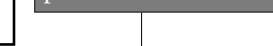


c) Para as unidades de saúde que não possuíam geolocalização, foi criado um script na linguagem $\mathrm{R}$ para pesquisa na ferramenta online Google $\mathrm{Maps}^{23}$, baseado nas informações de endereço disponíveis no cadastro CNES.

d) Para cada geolocalização resolvida na pesquisa do Google Maps foi realizado um processo de triagem e verificação baseado nos seguintes critérios: i) Para cidades de mais de 100.000 habitantes, verificou-se se a geolocalização resolvida pelo Google Maps refere-se, pelo menos, ao bairro designado pelo endereço, para as demais cidades, verificou-se se é referente à área do munícipio; ii) Para unidades rurais que não possuíam a geolocalização indicada na base do CNES, e que não puderam ser encontradas no mapa utilizando-se o Google Maps, foi realizada uma visita física ao local.

e) Das unidades localizadas, foram removidas as que se enquadrassem nos critérios de exclusão (Figura 1), restando 914 estabelecimentos (1,4\% das unidades foram removidas pelos critérios de exclusão). A lista de estabelecimentos encontrase publicada em http://rpubs.com/costa_/estabelecimentos.

Finalizada a coleta e preparação dos dados, foi iniciado o processo de montagem da rede relacional. O critério de construção dos relacionamentos foi a distância euclidiana do setor censitário com a geolocalização da unidade de saúde, excluindo os serviços/classificadores que não representassem serviços de uso pela população geral (Figura 1). Esta técnica de mensuração da acessibilidade geográfica é conhecida e bastante utilizada por apresentar uma abordagem simples e intuitiva, sendo baseada no cálculo da distância euclidiana entre o centro de massa do polígono ${ }^{24}$ que delimita o centro populacional e a localização geográfica do serviço de saúde, podendo ser utilizada outras medidas de custo de viagem e atribuindo-se diferentes impedâncias de deslocamento'.

Por este estudo operar em uma área urbana, onde a existência de um congestionamento de oferta de serviços similares entre diferentes unidades de saúde é um fenômeno esperado, o modelo de relacionamento assumiu uma faixa de tolerância de $20 \%$ em relação à distância mínima do relacionamento. Esta prática visou construir uma coleção de opções de acessibilidade geográfica para um determinado serviço que é oferecido por estabelecimentos de saúde com distâncias similares a um mesmo ponto de referência, aspecto debatido por Guagliardo ${ }^{9}$, como ponto de atenção da métrica de proximidade dos provedores mais próximos em áreas urbanas. $\mathrm{O}$ valor de tolerância é uma variável configurada no modelo, quanto maior o seu valor, maior a área de captura de serviços/classificadores similares na oferta potencial.

A distância euclidiana tem direta correlação com a distância percorrida na malha viária, contudo, este modelo pode ser considerado um preditor frágil da distância entre duas coordenadas geográficas. Jones et al. ${ }^{25}$ comparou as duas técnicas de determinação de distância demonstrando que em regiões urbanas esta diferença tende a se tornar menor que a encontrada em áreas rurais, especialmente em regiões com malha de ruas com reduzido impacto de acidentes geográficos. Fortney et al. ${ }^{21}$ também apresentam uma comparação de diferentes abordagens de medidas de acesso geográfico e destacam a similaridade, em termos de acurácia, da distância euclidiana e a distância obtida através de ferramentas de cálculo de navegação pela malha de estradas e rodovias através de processos heurísticos.Portanto, a utilização da distância euclidiana para regiões metropolitanas com reduzido impacto de acidentes geográfico sugere uma abordagem simples e com baixo custo computacional, técnica regularmente utilizada no campo de estudo de acesso geográfico na área de saúde ${ }^{26,27}$.

O resultado final da modelagem foi uma rede de setores censitários e estabelecimentos com 2.167.111 relacionamentos sob o critério de proximidade. A complexidade desta malha impede uma análise objetiva dos dados, desta maneira, a modelagem é conduzida para sua última etapa: a definição de um conjunto de relacionamentos que descrevem uma rede temática de análise e a aplicação das métricas de análise.

Para análise da rede este trabalho apoia-se na distância entre os vértices e a centralidade como ferramenta para avaliar a importância relativa dentro da estrutura modelada. Uma das mais básicas métricas utilizadas para descrever a importância de determinados vértices dentro de uma rede é o grau de centralidade. O grau de centralidade mede a importância relativa de um determinado vértice em função do seu número de relacionamentos com demais atores da rede $\mathrm{e}^{27}$. Traduzido para o contexto deste artigo, o grau de centralidade de um estabelecimento de saúde é refletido na quantidade de setores censitários que tem este estabelecimento como mais próximo na oferta potencial de um determinado serviço/classificador.

Como simplificação do modelo para aplicação inicial, cada setor censitário será representa- 
do com o peso de 1 (um) na contribuição geral da centralidade, aplicando-se assim um princípio de indiferença quanto a importância de cada setor na análise desta rede - embora cada setor possa variar em função de população, características socioeconômicas, culturais e epidemiológicas. O objetivo deste artigo se limita a apresentar as bases teóricas metodológicas de construção e análise da rede de oferta potencial de serviços de saúde utilizando a teoria de redes complexas.

Para o cálculo de centralidade optou-se pela utilização da métrica de PageRank ${ }^{28}$ por estabelecer um equilíbrio entre a oferta potencial e a disponibilidade do serviço. Duas abordagens de centralidade foram utilizadas, primeiro é aplicado sobre vértices individuais e, em seguida, para análise de grupos de vértices da rede, ou seja, que apresentem características comuns. A aplicação de métricas de centralidade para grupos em uma rede é abordado por Everett e Borgatti ${ }^{29}$ para métricas como Grau de Centralidade, Proximidade e Intermediação.Neste trabalho foi aplicado as formulações apresentadas por estes autores para a centralidade de PageRank. Desta forma, a centralidade por PageRank de um estabelecimento de saúde $p_{k}$ será dado por $C_{P R}\left(p_{k}\right)$ conforme a formulação:

$$
C_{P R}\left(p_{k}\right)=\sum_{i=1}^{n} a\left(p_{i}, p_{k}\right) \frac{C_{P R}\left(p_{i}\right)+\beta}{p_{i}^{\text {out }}}
$$

Sendo $a\left(p_{i}, p_{k}\right)=1$ se, e somente se o vértice $p_{i}$ (um setor censitário) apresentar um relacionamento com o vértice $p_{k}$ (um estabelecimento de saúde), do contrário o valor será 0 , por sua vez $C_{P R}\left(p_{i}\right)$ é a função de PageRank aplicada recursivamente ao nó $p_{i}$, o que, neste caso, será sempre 1 pois os setores censitários não recebem relacionamento, apresentando centralidade por PageRank igual a zero, e desta forma, é aplicado uma constante $\beta$ de valor 1 , representando o peso constante e indiferente deste setor. Em seguida dividimos a centralidade do peso $\beta$ pelo total de conexões de saída do setor censitário, representando aqui a situação em que um mesmo setor tem diferentes opções de acesso ao serviço em distâncias similares dentro do parâmetro de tolerância. Para o cálculo da centralidade de PageRank para grupo de vértices será usada a definição de Everett e Borgatti ${ }^{29}$ sumarizando o número de relacionamentos de $p_{i}$ para com $p_{k}$ sendo, neste caso, $p_{k} \mathrm{o}$ conjunto de estabelecimentos que se deseja medir a centralidade.

Para ilustrar a aplicação da metodologia aqui apresentada, será analisada uma rede constituída de serviços tipicamente ofertados por unidades de atenção básica. Assim, será possível refletir o arranjo estrutural adotado no modelo de atenção básico à saúde do ponto de vista da oferta de serviços de saúde no território da AMB.

\section{Resultado e discussão}

Para construção da rede para análise foram selecionados apenas serviços/classificadores oferecidos por estabelecimentos do tipo Posto de Saúde ou Centro de Saúde/Unidade Básica. Com base nos serviços oferecidos por estabelecimentos deste tipo, conforme cadastrado no CNES na competência de dezembro de 2018, foram selecionados os relacionamentos que apontam para este tipo de serviço/classificador, independentemente do tipo do estabelecimento que oferece esta modalidade de serviço. Portanto, o modelo não excluí unidades de nível hospitalar e ambulatorial que tenham serviços da natureza selecionada como parte de sua oferta.

Do ponto de vista estrutural, a rede foi constituída num formato bipartida, resultando numa coleção de 5.802 vértices do tipo setor censitário relacionando-se com 914 vértices do tipo estabelecimento de saúde, e multiplex, manifestado através de 73 tipos de relacionamento, sendo cada tipo um distinto serviço/classificador que caracteriza o tipo de serviço oferecido pelo estabelecimento, formando um total de 697.504 relacionamentos onde apresentam-se diferentes camadas de ofertas potenciais de redes de atenção à saúde. Dado o número de relacionamentos, a visualização da rede constitui-se uma tarefa bastante difícil, cabendo uma descrição numérica das suas características e métricas como ferramenta de análise.

Do ponto de vista da centralidade individual dos vértices do tipo estabelecimento de saúde (Tabela 1), foram isolados os 10 estabelecimentos com maior centralidade. Neste aspecto percebese um protagonismo dos hospitais, característica fundamentalmente explicada pela abrangente oferta de serviços de saúde e a localização em áreas de maior densidade demográfica. Contudo, é observado também, a existência de uma concentração de centralidade para alguns serviços específicos em algumas destas unidades. No Hospital Regional de Ceilândia, por exemplo, o serviço de "110 - Serviço de Atenção à Saúde Reprodutiva" concentra $35 \%$ de todos os relacionamentos possíveis para este tipo de serviço sendo $21 \%$ da demanda potencial tendo origem setores censitários dos municípios do entorno pertencentes a 
Tabela 1. Estabelecimentos de Saúde com maior centralidade.

\begin{tabular}{llccccc}
\hline \multirow{2}{*}{ CNES } & \multirow{2}{*}{ Estabelecimento de Saúde } & Distância & \multirow{2}{*}{ Média $(\mathbf{k m})$} & Absoluta & & \multicolumn{3}{c}{ Centralidade por PageRank } \\
\cline { 5 - 7 } & & 10,2 & 25.205 & 0,060 & $0,055 / 92$ & $0,005 / 08$ \\
\hline 0010480 & Hospital Regional de Ceilandia & 11,1 & 20.843 & 0,049 & $0,046 / 94$ & $0,003 / 06$ \\
0010499 & Hospital Regional de Taguatinga & 20,5 & 18.890 & 0,045 & $0,039 / 87$ & $0,006 / 13$ \\
0010537 & Hospital Materno Infantil de Brasília & 8,3 & 14.907 & 0,036 & $0,035 / 97$ & $0,001 / 03$ \\
2814897 & Hospital Regional do Guara & 14,2 & 13.612 & 0,033 & $0,031 / 94$ & $0,002 / 06$ \\
0010464 & Hospital Regional da Asa Norte & 19,7 & 12.813 & 0,031 & $0,026 / 84$ & $0,005 / 16$ \\
0011266 & UBS 02 Guara & 7,2 & 11.919 & 0,029 & $0,028 / 97$ & $0,001 / 03$ \\
2672197 & Hospital Regional de Samambaia & 11,1 & 11.211 & 0,027 & $0,023 / 85$ & $0,004 / 15$ \\
0010529 & Hospital Regional de Planaltina & 8,5 & 11.151 & 0,027 & $0,015 / 56$ & $0,012 / 44$ \\
5717515 & Hospital Regional de Santa Maria & 14,9 & 10.118 & 0,024 & $0,023 / 96$ & $0,001 / 04$ \\
\hline 265157 & Hospital da Região Leste & & & & DF/\% & GO/\% \\
\hline
\end{tabular}

Fonte: Elaborado pelos autores, 2019.

AMB. Entende-se "total de relacionamentos possíveis para este tipo de serviço", como forma de normalização da métrica de centralidade de $\mathrm{Pa}$ geRank, criando, portanto, um índice de centralidade relativo para cada vértice dentro da rede, desta maneira, uma unidade de saúde com 100\% de todos os relacionamentos possíveis representaria a única unidade oferecendo um dado serviço e, portanto, cobriria todos os relacionamentos oriundos da demanda potencial dos setores censitários.

Outra unidade que apresenta forte concentração em ofertas específicas é o Hospital Materno Infantil de Brasília. Nesta unidade observamos uma concentração $93 \%$ de todos os relacionamentos possíveis para o serviço de "139 - Serviço de Triagem Neonatal” (aqui inclui apenas o classificador "001 - Tratamento de Recém Nascido com Hipotireoidismo e Fenilcetonúria" por se encontrar entre a oferta de serviços oferecidos pela rede de unidades básicas), $31 \%$ de todos os relacionamentos possíveis para o serviço "110 - Serviço de Atenção à Saúde Reprodutiva" e $23 \%$ de todos os relacionamentos possíveis para o serviço "107 - Serviço de Atenção à Saúde Auditiva", com distância média entre os setores censitários relacionados e o estabelecimento de saúde onde o serviço é oferecido de 22, 25 e 27 quilômetros, respectivamente. Por último, uma acentuada centralidade foi encontrada na unidade UBS 02 Guará com o serviço "134 - Serviço de Práticas Integrativas e Complementares" com $34 \%$ de todos os relacionamentos possíveis e distância média de 20 quilômetros.

Quando observamos a centralidade sob a perspectiva do agrupamento dos estabelecimentos em função de sua classificação no CNES por tipo de unidade (Tabela 2), notamos, mais uma vez, o protagonismo dos hospitais como principais centralizadores da oferta potencial de serviços com $45 \%$ de todos os relacionamentos possíveis dentro da rede temática de serviços oferecidos por unidades de atenção básica. Portanto, embora os estabelecimentos de saúde do tipo Centro de Saúde/Unidade Básica apresentem maior capilaridade e implementação descentralizada, este tipo de unidade responde apenas por $24 \%$ da demanda potencial, o que significa que, neste modelo, os hospitais capturam grande parte da demanda por estarem mais próximos ou por estarem entre as poucas unidades que ofertam os serviços selecionados para montagem desta rede. É importante notar que as unidades do tipo "Centro de Saúde/Unidade Básica" respondem por mais de $95 \%$ de todos os relacionamentos quando o serviço é "101 - Estratégia de Saúde da Família" e "147 - Serviço de Apoio à Saúde da Família", com distância média de 6,6 e 6,4 quilômetros respectivamente. Embora as Unidades Básicas respondam apenas por 35\% dos relacionamentos para o serviço "112 - Serviço de Atenção ao Pré-Natal, Parto e Nascimento", observamos mais de $80 \%$ de concentração quando tratamos especificamente do classificador "001 - Acompanhamento do Pré-Natal de Risco Habitual", com distância média de 6 quilômetros.

É possível observar algumas potenciais falhas de cobertura ou um indicativo de políticas públicas em fase de implantação, por exemplo, os serviços "134 - Práticas Integrativas e Complementares", "158 - Serviço de Atenção Integral a Hanseníase" e "165 - Atenção às Pessoas em Situação de Violência Sexual" embora respondam por mais de $65 \%$ dos relacionamentos na rede 
Tabela 2. Centralidade Agrupada por Tipo de Unidade.

\begin{tabular}{|c|c|c|c|c|c|c|}
\hline \multirow[b]{2}{*}{ Tipo do Estabelecimento } & \multirow{2}{*}{$\begin{array}{c}\text { Total } \\
\text { Estabelecimentos }\end{array}$} & \multirow{2}{*}{$\begin{array}{l}\text { Distância } \\
\text { Média } \\
(\mathbf{k m})\end{array}$} & \multicolumn{4}{|c|}{ Centralidade do Grupo porPageRank } \\
\hline & & & Absoluta & Normalizada & $\mathrm{DF} / \%$ & GO/\% \\
\hline Hospital Geral & 50 & 12,4 & 189.806 & 0,448 & $0,381 / 85$ & $0,067 / 15$ \\
\hline Centro de Saúde/Unidade Básica & 484 & 16,2 & 99.791 & 0,236 & $0,178 / 75$ & $0,058 / 25$ \\
\hline Clínica/Centro de Especialidade & 87 & 14,0 & 32.285 & 0,076 & $0,041 / 53$ & $0,036 / 47$ \\
\hline Policlínica & 54 & 12,9 & 29.535 & 0,070 & $0,056 / 80$ & $0,014 / 20$ \\
\hline $\begin{array}{l}\text { Unidade de Apoio Diagnose e } \\
\text { Terapia }\end{array}$ & 67 & 9,5 & 19.426 & 0,046 & $0,024 / 52$ & $0,022 / 48$ \\
\hline Pronto Atendimento & 14 & 7,2 & 16.512 & 0,039 & $0,032 / 80$ & $0,008 / 20$ \\
\hline Hospital Especializado & 9 & 14,4 & 9.123 & 0,022 & $0,019 / 86$ & $0,003 / 14$ \\
\hline Farmácia & 18 & 17,0 & 7.752 & 0,018 & $0,013 / 68$ & $0,006 / 32$ \\
\hline Polo Academia da Saúde & 16 & 34,1 & 4.302 & 0,010 & $0,008 / 80$ & $0,002 / 20$ \\
\hline Hospital/Dia - Isolado & 4 & 12,1 & 4.179 & 0,010 & $0,005 / 50$ & $0,005 / 50$ \\
\hline Outros & 111 & 15,8 & 10.767 & 0,025 & $0,012 / 48$ & $0,013 / 52$ \\
\hline
\end{tabular}

Fonte: Elaborado pelos autores, 2019.

de Unidades Básicas, estes serviços apresentam distância média superior a 20 quilômetros, potencialmente indicando uma baixa oferta na rede de saúde de forma geral e uma baixa cobertura na atenção básica.

Quando analisamos a distribuição dos principais serviços que compõe a oferta potencial das unidades do tipo "Hospital Geral" observamos uma concentração de mais de $80 \%$ dos relacionamentos possíveis na rede para os seguintes serviços/classificadores: "110 - Serviço de Atenção à Saúde Reprodutiva/001 - Atenção a Infertilidade" (98\% dos relacionamentos, distância média $21 \mathrm{Km})$, “139 - Serviço de Triagem Neonatal/001 - Tratamento Recém Nascido com Hipotireoidismo e Fenilcetonúria” (94\% dos relacionamentos, distância média $25 \mathrm{Km}$ ), "112 - Serviço de Atenção ao Pré-Natal, Parto e Nascimento/003 - Gestação em Risco Habitual” (92\% dos relacionamentos, distância média 19 Km), "113 - Serviço de Atenção Domiciliar/002 - Internação Domiciliar” (84\% dos Relacionamentos, distância média $10 \mathrm{Km})$, "140 - Serviço de Urgência e Emergência/019 - Pronto Socorro Geral/Clínico" (83\% dos relacionamentos, distância média $8 \mathrm{Km}$ ) e "135 - Serviço de Reabilitação/004 - Reabilitação Visual, Mental, Múltiplas Deficiências” (80\% dos relacionamentos, distância média $24 \mathrm{Km}$ ).

A perspectiva agrupada permite entender a distribuição dos relacionamentos potenciais em função da relação do tipo de serviço com o tipo da unidade, destacando assim como a oferta potencial de serviços é constituída dentro do espaço geográfico da AMB. O Gráfico 1 ilustra esta distribuição destacando serviços tais como: Diagnóstico por Laboratório Clínico, Urgência e Emergência, Triagem Neonatal, Reabilitação, Fisioterapia, Diagnostico por Métodos Dinâmicos, Diagnóstico por Imagem, Diagnóstico por Anatomia Patológica e/ou Citopato, Atenção Domiciliar, Atenção ao Pré-Natal/Parto/Nascimento, Atenção à Saúde Reprodutiva, Atenção à Saúde do Trabalhador e Atenção à Saúde Auditiva, tem mais de $50 \%$ de sua oferta potencial coberta por estabelecimentos hospitalares quando observamos a dimensão da acessibilidade geográfica à estes serviços, denotando, portando, uma acessibilidade que favorece a centralização destes serviços em unidades de maior densidade de atendimento e menor grau de regionalização. Por outro lado, observamos que os serviços: Atenção às Pessoas em Situação de Violência Sexual, Serviço de Atenção Integral em Hanseníase, Laboratório de Prótese Dentária, Coleta de Materiais Biológicos, Práticas Integrativas e Complementares, Dispensação de Órteses/Próteses/Materiais Especiais, Atenção ao Paciente com Tuberculose e Estratégia da Saúde da Família, tem sua oferta potencial em grande parte representada por unidades do tipo Centro de Saúde/Unidade Básica.

Embora os resultados sugerem uma centralização da oferta potencial em alguns hospitais, característica que denota ausência do serviço na plataforma capilar e descentralizada de unidades básicas, ainda assim, observamos que vários dos serviços encontram em uma faixa de distância média inferior a 10 quilômetros da demanda potencial (Gráfico 2), havendo uma distância mé- 


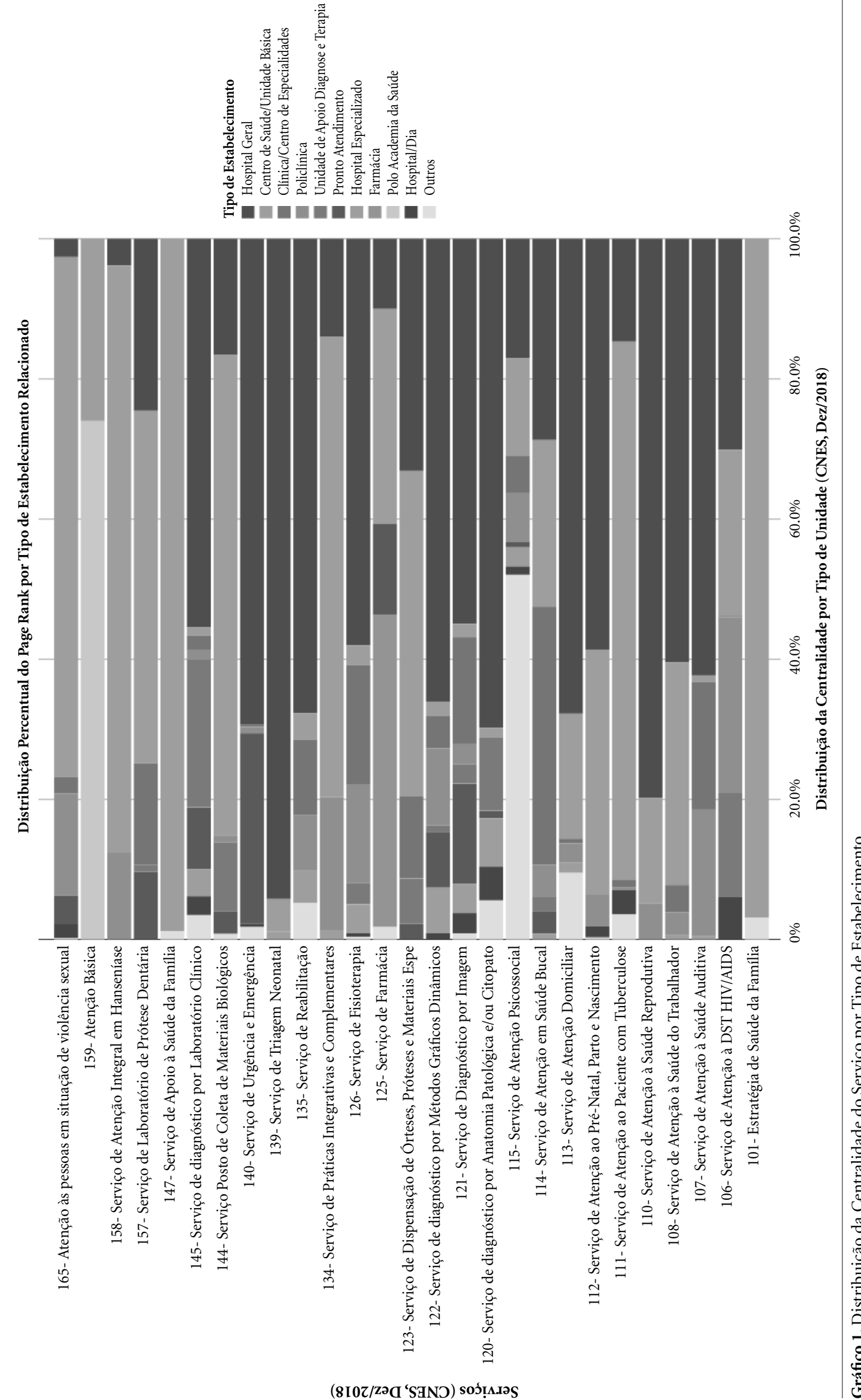

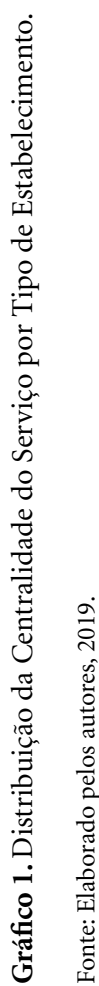




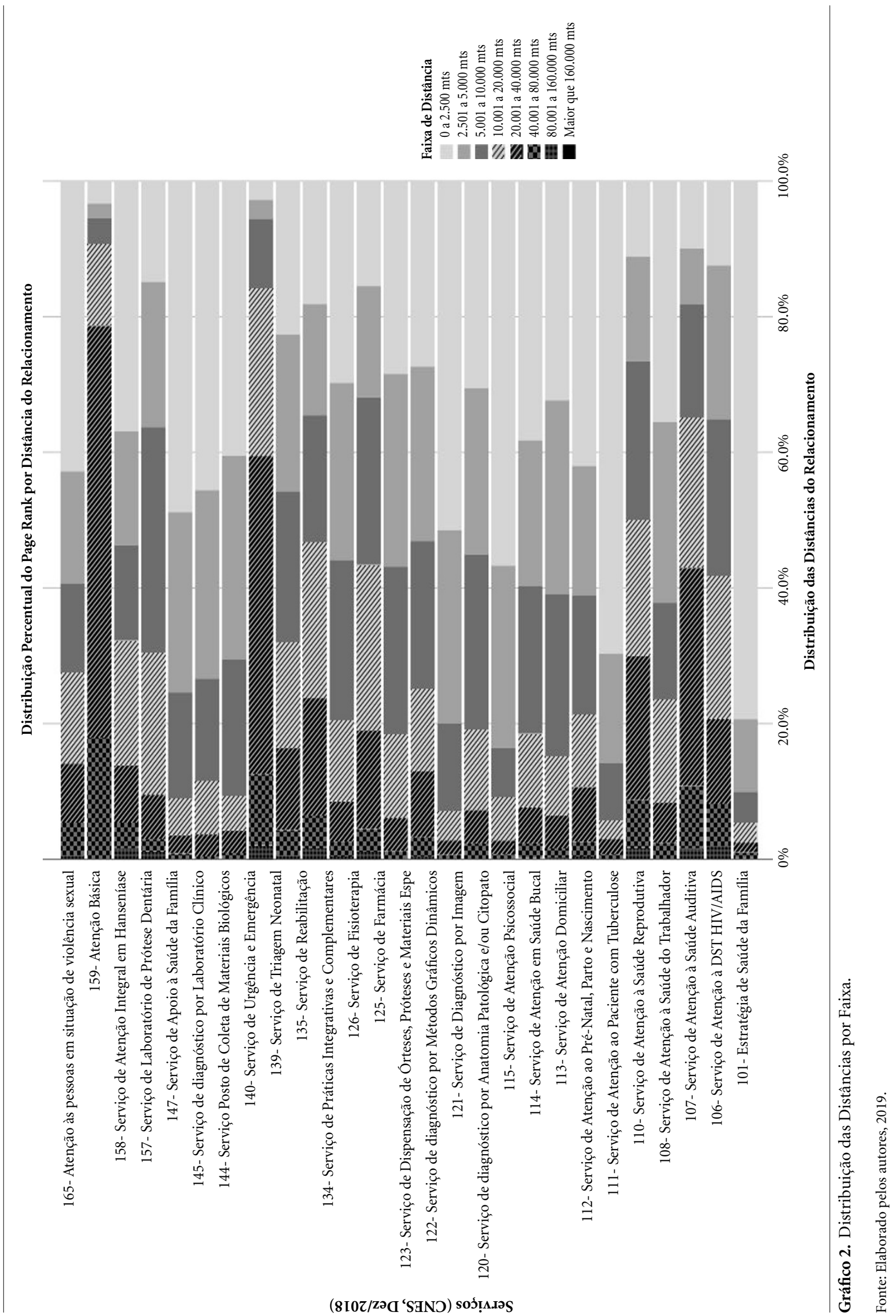


dia maior para serviços como: Atenção Integral a Hanseníase $(31 \mathrm{Km})$, Triagem Neonatal $(28 \mathrm{Km})$, Atenção à Saúde Auditiva $(25 \mathrm{Km})$, Atenção à DST/HIV/AIDS $(21 \mathrm{Km})$, Práticas Integrativas e Complementares $(20 \mathrm{Km})$, Atenção à Saúde Reprodutiva $(20 \mathrm{Km})$, Atenção à Pessoas em Situação de Violência Sexual (19 Km) e Reabilitação $(16 \mathrm{Km})$, todos os demais serviços apresentam distância média inferior a 16 quilômetros. Embora o serviço “159 - Atenção Básica” apresente uma distância média de 33 quilômetros. Esta aparente anomalia pode ser explicada pelo fato da portaria que normaliza esta classificação no CNES ter sido publicada apenas em janeiro de $2019^{30}$.

É importante notar que o resultado de maior centralização em unidades de nível hospitalar não reflete uma disfunção na implementação de políticas voltadas para atenção básica. Este é um fenômeno natural para os serviços selecionados para análise, visto que todos os setores censitários estabelecem relacionamentos com todos os tipos de serviço, independentemente da demanda oriunda do perfil epidemiológico destes setores. Contudo, o Gráfico 1 nos oferece uma ideia de como cada serviço será distribuído dentro da oferta potencial das unidades e considerando a menor distância percorrida. Esta métrica é um bom indicativo se um determinado tipo de serviço está sofrendo uma influência de falhas de cobertura. Um exemplo que poderia indicar boa cobertura potencial da atenção básica está refletida no serviço "111 - Serviço de Atenção ao Paciente com Tuberculose", onde observamos uma cobertura potencial majoritariamente promovida por unidades básicas (Centralidade de 75\%, Gráfico 1) e distância média com mais de $80 \%$ dos relacionamentos com até 5 quilômetros de distância.

Embora o conceito de acesso à saúde implique um complexo encadeamento de dimensões para análise dos determinantes do uso dos serviços de saúde ${ }^{31}$, o acesso geográfico é fator chave para compreensão das decisões que levam o indivíduo estabelecer relações de cuidado com sua própria saúde e estar situado no processo de cuidado contínuo e latitudinal da atenção básica. Higgs ${ }^{26}$ destaca o efeito da distância sobre as baixas taxas de utilização dos serviços de saúde, implicando uma inversa relação entre distância e a taxa de utilização, contudo o autor destaca que esta relação pode variar em função da natureza do serviço utilizado. Fortney et al. ${ }^{32}$, destacam a correlação entre acessibilidade geográfica e a continuidade no tratamento da depressão, campo que sugere oportunidades de estudo aplican- do este modelo utilizando um recorte da rede temática de atenção psicossocial. Rocha et al. ${ }^{33}$ estudam a acessibilidade geográfica de serviços de urgência e emergência através de um recorte nacional demonstrando a relação entre unidades e municípios, outra perspectiva que é oportuna para análise no contexto espacial de setores censitários, permitindo observar lacunas assistenciais dentro dos municípios.

Por estarmos representando um recorte da realidade através de um modelo, é importante reconhecer algumas limitações. Delamater et al. ${ }^{34}$, discutem a respeito de como métodos de avaliação de acessibilidade geográfica são submetidos a simplificações, necessárias e inerentes ao processo de modelagem, mas que devem ser claramente reconhecidas. Para o modelo sugerido aqui, simplificações foram assumidas no que se refere a uniformização da impedância de deslocamento e indiferença quanto o modal de transporte. Desta maneira, assume-se a distância euclidiana como uma aproximação para o esforço de deslocamento entre os pontos mapeados, independentemente de qualquer outra variável como trânsito, tipo de estrada ou meio de transporte.

O processo de construção de um modelo implica em decisões de modelagem que configuram a realidade estudada. Este processo de decisão, que aqui demos o nome de "definição da rede temática”, implica na capacidade de analisar diferentes arranjos do sistema de saúde, podendo dar apoio à criação de métricas especializadas para monitoração de diferentes políticas públicas e redes de atenção à saúde. No exemplo de análise demonstramos a aplicação ao selecionar serviços que estão disponíveis entre as unidades básicas da $\mathrm{AMB}$, embora não seja objetivo desta análise servir de instrumento de avaliação da cobertura da atenção básica neste território pudemos observar como tais serviços estão distribuídos, tanto do ponto de vista espacial, como do ponto de vista da oferta por tipo de estabelecimento, dados que, quando triangulados com outros instrumentos metodológicos podem orientar a análise da implementação de políticas públicas relacionadas à atenção básica.

Por fim, percebe-se que as informações demográficas e sociais contidas nos dados agregados do CENSO e as informações de recursos de saúde mantidas no CNES conferem uma ampla plataforma de análise para compreensão dos arranjos estruturais e organizacionais dos fatores capacitantes e predisponentes, tais como apresentados por Andersen ${ }^{4}$, Penchansky e Thomas ${ }^{5}$. Futuras aplicações do modelo poderão incluir a 
visão do acesso realizado observando os procedimentos executados por cada unidade e comparando com a centralidade das unidades de saúde ponderada pela população origem da demanda potencial, tal visão poderia auxiliar na identificação de desequilíbrios na cobertura dos serviços e indicar possibilidade da existência de outras barreiras para acesso à saúde.Sob esta ótica, há ainda muito trabalho a ser feito no aprofundamento do estudo das relações espaciais observando a distribuição de serviços e recursos de saúde tendo foco a equidade social destes arranjos.

\section{Colaboradores}

DRT Costa: concepção e delineamento da pesquisa, redação do artigo, análise e interpretação dos dados. JOM Barreto: concepção e delineamento da pesquisa, revisão crítica e aportes teóricos e aprovação da versão final. RB Sampaio: revisão crítica e aportes teóricos e aprovação da versão final. 


\section{Referências}

1. Mendes EV. As redes de atenção à Saúde. 2a ed. Brasília: OPAS; 2011.

2. Brasil. Ministério da Saúde (MS). Portaria no 2.436, de 21 de setembro de 2017. Aprova a Política Nacional de Atenção Básica, estabelecendo a revisão de diretrizes para a organização da Atenção Básica, no âmbito do Sistema Único de Saúde (SUS). Diário Oficial da União; 2017.

3. Brasil. Ministério da Saúde (MS). Resolução no ${ }^{\circ}$, de 29 de setembro de 2011. Estabelece diretrizes gerais para a instituição de Regiões de Saúde no âmbito do Sistema Único de Saúde (SUS), nos termos do Decreto No 7.508, de 28 de junho de 2011. Diário Oficial da União; 2011.

4. Andersen RM. Revisiting the Behavioral Model and Access to Medical Care: Does It Matter? J Health Soc Behav 1995; 36(1):1-10.

5. Penchansky R, Thomas JW. The concept of access definition and relationship to consumer satisfaction. Med Care 1981; 19(2):127-140.

6. Huerta Munoz U, Källestål C. Geographical accessibility and spatial coverage modeling of the primary health care network in the Western Province of Rwanda. Int J Health Geogr 2012; 11:40.

7. Abbas AA, Walker GJ. Determinants of the utilization of maternal and child health services in Jordan Int $J$ Epidemiol 1986; 15(3):404-407.

8. Feikin DR, Nguyen LM, Adazu K, Ombok M, Audi A, Slutsker L, Lindblade KA. The impact of distance of residence from a peripheral health facility on pediatric health utilisation in rural western Kenya. Trop Med Int Health 2009; 14(1):54-61.

9. Guagliardo MF. Spatial accessibility of primary care: concepts, methods and challenges. Int J Health Geogr 2004; 3(1):3.

10. Companhia de Planejamento do Distrito Federal (CODEPLAN). Delimitação do Espaço Metropolitano de Brasília (Área Metropolitana de Brasília). Nota Técnica no 1/2014. Brasília: CODEPLAN; 2014.

11. Brasil. Ministério da Saúde (MS). Departamento de Informática do SUS. CNES, Cadastro Nacional de Estabelecimentos de Saúde [Intermet]. [acessado 2018 abr 12]. Disponível em http://cnes.datasus.gov.br/ pages/sobre/institucional.jsp.

12. Instituto Brasileiro de Geografia e Estatística (IBGE). Guia do Censo [Internet]. [acessado 2018 abr 12]. Disponível em https://censo2010.ibge.gov.br/materiais/ guia-do-censo/operacao-censitaria.html.

13. Freman LC. Turning a profit from mathematics: The case of social networks. J Math Sociol 1984; 10(34):343-360.

14. Wasserman S, Faust K. Social Network Analysis Methods and Applications. Cambridge: Cambridge University Press; 1994.

15. Newman MEJ. Networks: An Introduction. Oxford: Oxford University Press; 2010.

16. Wilson TP. Relational networks: An extension of sociometric concepts. Soc Networks 1982; 4(2):105-116.

17. Borgatti SP, Everett MG. Network analysis of 2-mode data. Soc Networks 1997; 19(3):243-269.
18. Boccaletti S, Bianconi G, Criado R, Del Genio CI, Gómez-Gardeñes J, Romance M, Sendiña-Nadaj I, Wang $\mathrm{Z}$, Zanin $\mathrm{M}$. The structure and dynamics of multilayer networks. Physics Reports 2014; 544(1):1-122.

19. RStudio. Team RStudio: Integrated Development for $R$ [Internet]. Boston: RStudio, Inc; 2015. [acessado 2018 abr 12]. Disponível em: https://www.rstudio.com/.

20. Bastian M, Heymann S, Jacomy M. Gephi: an open source software for exploring and manipulating networks. International AAAI Conference on Weblogs and Social Media; 2009.

21. Fortney J, Rost K, Warren J. Comparing Alternative Methods of Measuring Geographic Access to Health Services. Health Serv Outcomes Res Methodol 2000; 1(2):173-184.

22. Secretaria de Estado de Saúde do Distrito Federal. Mapa das Unidades de Saúde [Internet]. [acessado 2019 fev 20]. Disponível em: https://salasit.saude.df. gov.br/mapa-unidades-de-saude/.

23. Google Maps. Google Maps Directions API [Internet]. [acessado 2019 fev 20]. Disponível em: https://developers.google.com/maps/documentation/directions/ start.

24. Bourke P. Calculating the Area and Centroid of Polygon [Internet]. 1988 [acessado 2019 set 10]. Disponível em: https://www.seas.upenn.edu/ sys502/extra_materials/Polygon\%20Area\%20and\%20Centroid.pdf.

25. Jones SG, Ashby AJ, Momin SR, Naidoo A. Spacial Implications Associated with Using Euclidean Distance Measurements and Geographic Centroid Imputation in Health Care Research. Health Serv Res 2010; 45(1):316-327.

26. Higgs G. The role of GIS for health utilization studies: literature review. Health Serv Outcomes Res Methodol 2009; 9(2):84-99.

27. Freman LC. Centrality in Social Network Conceptual Clarification. Soc Networks 1978; 1(3):215-239.

28. Page L, Brin S, Motwani R, Winograd T. The PageRank Citation Ranking: Bringing Order to the Web. Technical Report. Stanford InfoLab; 1999 [acessado 2019 jun 10]. Disponível em: http://ilpubs.stanford. edu:8090/422/1/1999-66.pdf.

29. Everett MG, Borgatti SP. The Centrality of Groups and Classes. J Math Sociol 1999; 23(3):181-201.

30. Brasil. Ministério da Saúde (MS). Secretaria de Atenção à Saúde. Portaria no 18, de 7 de janeiro de 2019. Estabelece regras para o cadastramento das equipes da Atenção Básica no Cadastro Nacional de Estabelecimentos de Saúde (CNES), conforme diretrizes da Política Nacional de Atenção Básica. Diário Oficial da União; 2019.

31. Wheat B. Patterns of Utilization: The Patient Perspective. Med Care 1985; 23(5):438-460.

32. Fortney J, Rost K, Zhang M, Warren J. The Impact of Geographic Accessibility on The Intensity and Quality of Depression Treatment. Med Care 1999; 37(9):884893. 
33. Rocha TAH, Silva NC, Amaral PV, Barbosa ACQ, Rocha JVM, Alvares V, Almeida DG, Thumé E, Thomaz EBAF, Queiroz RCS, Souza MR, Lein A, Lopes DP, Staton CA, Vissoci JRN, Facchini LA. Addressing geographic access barriers to emergency care services: A national ecologic study of hospitals in Brazil. Int $J$ Equity Health 2017; 16(1):149.

34. Delamater PL, Messina JP, Shortridge AM, Grady SC. Measuring geographic access to health care: raster and network-based methods. Int J Health Geogr 2012; 11(1):15.

Artigo apresentado em 12/10/2019

Aprovado em 26/04/2020

Versão final apresentada em 28/04/2020

Editores-chefes: Romeu Gomes, Antônio Augusto Moura da Silva 